\title{
Cognitive, EEG, and MRI features of COVID-19 survivors: a 10-month study
}

\author{
Giordano Cecchetti ${ }^{1,2,3,5}$ - Federica Agosta ${ }^{1,2,5}$ - Elisa Canu' ${ }^{1} \cdot$ Silvia Basaia $^{1}(\mathbb{C}) \cdot$ Alessandra Barbieri $^{2}$.

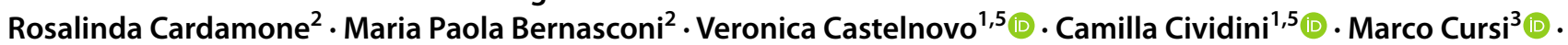

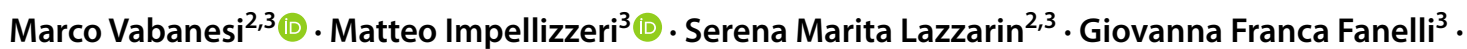

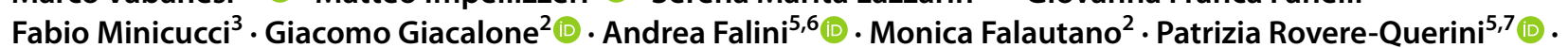 \\ Luisa Roveri' ${ }^{2}$. Massimo Filippi ${ }^{1,2,3,4,5}$
}

Received: 31 January 2022 / Revised: 21 February 2022 / Accepted: 22 February 2022 / Published online: 6 March 2022

(c) The Author(s), under exclusive licence to Springer-Verlag GmbH Germany 2022

\begin{abstract}
Background and objectives To explore cognitive, EEG, and MRI features in COVID-19 survivors up to 10 months after hospital discharge.

Methods Adult patients with a recent diagnosis of COVID-19 and reporting subsequent cognitive complaints underwent neuropsychological assessment and 19-channel-EEG within 2 months (baseline, $N=49$ ) and 10 months (follow-up, $N=33$ ) after hospital discharge. A brain MRI was obtained for 36 patients at baseline. Matched healthy controls were included. Using eLORETA, EEG regional current densities and linear lagged connectivity values were estimated. Total brain and white matter hyperintensities (WMH) volumes were measured. Clinical and instrumental data were evaluated between patients and controls at baseline, and within patient whole group and with/without dysgeusia/hyposmia subgroups over time. Correlations among findings at each timepoint were computed.

Results At baseline, 53\% and 28\% of patients showed cognitive and psychopathological disturbances, respectively, with executive dysfunctions correlating with acute-phase respiratory distress. Compared to healthy controls, patients also showed higher regional current density and connectivity at delta band, correlating with executive performances, and greater WMH load, correlating with verbal memory deficits. A reduction of cognitive impairment and delta band EEG connectivity were observed over time, while psychopathological symptoms persisted. Patients with acute dysgeusia/hyposmia showed lower improvement at memory tests than those without. Lower EEG delta band at baseline predicted worse cognitive functioning at follow-up.

Discussion COVID-19 patients showed interrelated cognitive, EEG, and MRI abnormalities 2 months after hospital discharge. Cognitive and EEG findings improved at 10 months. Dysgeusia and hyposmia during acute COVID-19 were related with increased vulnerability in memory functions over time.
\end{abstract}

Keywords COVID-19 $\cdot$ Long COVID $\cdot$ MRI $\cdot$ EEG $\cdot$ Cognitive disturbances

Massimo Filippi

filippi.massimo@hsr.it

1 Neuroimaging Research Unit, Division of Neuroscience, IRCCS San Raffaele Scientific Institute, Via Olgettina, 60, 20132 Milan, Italy

2 Neurology Unit, IRCCS San Raffaele Scientific Institute, Via Olgettina, 60, 20132 Milan, Italy

3 Neurophysiology Service, IRCCS San Raffaele Scientific Institute, Via Olgettina, 60, 20132 Milan, Italy
4 Neurorehabilitation Unit, IRCCS San Raffaele Scientific Institute, Via Olgettina, 60, 20132 Milan, Italy

5 Vita-Salute San Raffaele University, Via Olgettina, 60, 20132 Milan, Italy

6 Department of Neuroradiology, IRCCS San Raffaele Scientific Institute, Milan, Italy

7 Internal Medicine, Diabetes and Endocrinology Unit, IRCCS San Raffaele Scientific Institute, Milan, Italy 


\section{Introduction}

The heterogeneity of acute clinical presentation of coronavirus disease 2019 (COVID-19) and related pathogenic mechanisms have been largely explored in the past year [1]. More recently, increasing awareness of long lasting and multi-organ effects of severe acute respiratory syndrome coronavirus 2 (SARS-CoV-2) have raised concerns about their duration and reversibility [2].

Among others, reports of neurological sequelae in COVID-19 survivors include chronic malaise, diffuse myalgia, sleep disturbances, migraine-like headaches, ageusia, anosmia, and cognitive impairment in a significant proportion of patients [2,3]. Specifically, cognitive impairment in COVID-19 survivors has been amply described with observations from 1 to 6 months after infection recovery [4-9]. In two longitudinal studies, altered cognitive performances were reported in $81 \%$ and in $21 \%$ of patients, at 3- and 6-months follow-up, respectively $[8,9]$. However, up to date only few studies have explored cognitive involvement through structured neuropsychological assessments, with the most observing a short follow-up and involving only few patients. Individuals with previous COVID-19 also experience a wide range of persistent psychiatric disturbances including post-traumatic stress disorder (PTSD), depression and anxiety [8, $10,11]$. Psychiatric sequelae have been reported in $56 \%$ of patients in a cohort of 402 COVID-19 survivors at 1 month after hospitalization in Italy, and in almost $25 \%$ of patients at 6 months follow-up in China [12-14].

Proposed pathogenic contributors to neurocognitive and psychiatric involvement in 'Post-acute COVID-19 syndrome' might be direct cerebral viral invasion and cellular damage, systemic inflammation with cytokine release and cerebral microvascular changes [15].

Concerning brain alterations, a recent British MRI study of 394 patients with available scans before and after COVID-19 revealed a loss of grey matter (GM) in limbic cortical areas directly linked to the primary olfactory and gustatory systems, possible hallmark of the cerebral spread of the virus [16]. Interestingly, in a recent German study involving 26 COVID-19-patients, $69 \%$ of them showed cognitive impairment immediately after infection resolution, and two-thirds exhibited a predominant frontoparietal hypometabolism at brain [18F]FDG-PET [17]. Since neuropathology excluded irreversible neocortical damage and highlighted a pronounced microglia activation within white matter (WM), cortical hypometabolism has been interpreted as a consequence of remote WM or brainstem damage [17]. Moreover, in our previous EEG study on 18 acute COVID-19 patients, we suggested that EEG alterations might represent a useful tool to evaluate early cerebral involvement in COVID-19; a frequent finding in our cohort was an anterior prevalence of slow waves, correlating with metabolic and hypoxic alterations [18-20]. None of the studies available in the literature focused on EEG analysis after infection resolution.

Considering the lack of longitudinal studies with long follow-up and with structured neuropsychological and EEG assessments, we aimed at exploring longitudinal cognitive and concomitant EEG features in a population of adult COVID-19 survivors up to 10 months after hospital discharge. Brain MRI at study entry was also performed to investigate possible atrophic and/or vascular brain alterations after COVID-19.

\section{Methods}

\section{Participants and study procedures}

Forty-nine consecutive adult patients recovered from COVID-19 were recruited between April and May 2020 among those with recent ( $30 \pm 15$ days) admission to Emergency Room (ER) due to respiratory symptoms at the IRCCS San Raffaele Scientific Institute, Milan, Italy. Patients were recruited among 201 subjects evaluated at the 1-month postdischarge neurological examination, comprehensive of past and recent medical history collection from medical records. In particular, cardiovascular risk factors, pre-existing medical conditions, length of hospital stay, acute COVID19-related neurological symptoms, mechanical ventilation need and type, and National Early Warning Score (NEWS) [21] at ER admission were collected. Eligibility required: a diagnosis of SARS-CoV-2 infection confirmed through a positive reverse transcription-polymerase chain reaction (RT-PCR) nasopharyngeal swab, a subsequent recovery from acute illness with functional independence (modified Rankin Scale <2) [22, 23], fluency in Italian language, and new-onset self-reported cognitive disturbances. At the time of recruitment, all patients had a negative SARS-CoV-2 RTPCR test.

As part of the research protocol, clinical evaluation, neuropsychological testing battery, and 19-channel EEG were acquired within 2 months (49/49 patients-baseline) and within 10 months (33/49 patients-follow-up) after hospital discharge. Thirty-six out of 49 participants also underwent a $3 \mathrm{~T}$ brain MRI at baseline only.

Two different groups of age- and sex-matched healthy controls, with no history of cognitive impairment or of any other neurological disease, were retrospectively selected for the cognitive and MRI (36 subjects) analyses and for the EEG (33 subjects) analyses among those recruited by the Neuroimaging Research Unit and the Neurophysiology service, IRCCS San Raffaele Scientific Institute, respectively, 
between May 2017 and April 2020. For controls' selection, an "exact" matching analysis was used, in which each 'patient' was matched to a '(cognitive/MRI or EEG) control' with exactly the same age and sex (MatchIt package-R Statistical Software; version 4.0.3; R Foundation for Statistical Computing, Vienna, Austria). A comprehensive neuropsychological battery was obtained for each subject of the cognitive/MRI control group, whereas no cognitive assessments were acquired for the EEG healthy controls. Collection of past medical history for both control groups, comprehensive of cardiovascular risk factors and pre-existing medical conditions, was made by phone calls. Data of healthy controls were available at baseline only.

Exclusion criteria for all subjects were: medical illnesses or substance abuse that could interfere with cognitive functioning and EEG electrical activity; any (other) major systemic, psychiatric, or neurological illnesses; and other causes of focal or diffuse brain damage at routine MRI.

\section{Cognitive study}

Experienced neuropsychologists blinded to EEG and MRI results performed neuropsychological assessments. A battery of tests investigating the main cognitive domains (global cognition, executive functions, memory, visuospatial functions, language) was implemented for patients and healthy controls (Table e-1). At each timepoint, the presence of cognitive impairment was defined by a performance lower than the normative values (available for all tests administered to patients) in at least two tests within the same domain.

The presence of neuropsychiatric symptoms suggestive of depression or PTSD was defined from patients self-reports and unstructured interviews.

\section{EEG study}

\section{EEG acquisition}

Standard EEGs were acquired during the morning and in resting awake condition, under control of vigilance, on a computer-based system (Micromed System PLUS, Version 1.04, Micromed S.p.A., Mogliano Veneto, Italy) from 19 standard 10/20 electrode locations with linked ear reference [24]. EEG acquisition and pre-processing were previously described $[25,26]$. In particular, as last step of preprocessing, an independent component analysis (ICA) was performed to remove stereotyped eye, muscle and line noise (EEGLAB toolbox within Matlab; version 2020.0) [27, 28].

\section{Individual alpha frequency}

The occipital power spectrum was obtained for each subject by averaging the spectra of occipital channels (i.e., $\mathrm{O} 1$ and $\mathrm{O} 2$ ); the frequency within the extended alpha range $(7-13 \mathrm{~Hz})$ showing a power peak in the occipital power spectrum was the individual alpha frequency (IAF) [25].

\section{Current source density analysis}

Source estimation analysis was previously described [25, 26]. Experienced observers, blinded to participants' identity, performed the analyses. Briefly, to estimate cortical current source densities (CSD) exact low-resolution brain electromagnetic tomography (eLORETA) was used [29], which assesses current densities at 6239 voxels with zero error localization within the cortical GM of a realistic head model coregistered to the MNI and Talairach human brain atlases. EEG inverse solutions were estimated at 7 frequency bands for each subject (i.e., delta, theta, alpha1, alpha2, beta1, beta2, beta3) and subsequently normalized with the eLORETA total power within each of the six regions of interest (ROI-bilateral frontal, central-temporal, and parieto-occipital regions; see Table e-2) [25, 26] obtained by averaging across all frequencies and all voxels of the ROI. Normalized values were then averaged for all voxels belonging to each ROI by means of a homemade Matlab routine (R2017a 9.2, Mathworks, Natick, MA).

\section{Connectivity analysis}

To explore EEG connectivity, linear lagged connectivity (LLC), which measures the similarity between signals in the frequency domain, was computed using eLORETA among each pair of ROI. Advanced graph theoretical methods were applied, in which the functional network can be represented as a graph, consisting of nodes (brain regions), and edges (or connections) between regions that are functionally linked. The theoretical and methodological approach for the EEG network reconstruction was recently described [26]. Briefly, undirected, weighted graphs were obtained by computing LLC among each pair of ROI, in both groups of patients and matched healthy controls. Mean multivariate time series were extracted from each ROI by averaging the signal from all voxels within each region. Subsequently, the LLC measure between the mean time series of each ROI pair, indicating the level of EEG connectivity between regions $i$ and $j$, was entered into cell $\mathrm{c}(i, j)$ of a matrix. LLC values are non-negative, and take the value zero only when there is independence between ROI time series [30].

\section{MRI study}

\section{MRI acquisition}

MR sequences acquired using a 3T MR scanner are reported in Table e-3. 


\section{Brain tissue volumes}

Brain tissue total volume, GM and WM volumes, normalized for subject head size, were estimated with SIENAX (part of FSL 5.0.9) [31, 32]. SIENAX starts by extracting brain and skull images from the single whole-head input data. The brain image is then affine-registered to MNI152 space (using the skull image to determine the registration scaling); this is primarily to obtain the volumetric scaling factor, to be used as a normalization for head size. Next, tissue-type segmentation with partial volume estimation is carried out to calculate total volume of brain tissue (including separate estimates of volumes of GM and WM).

\section{White matter hyperintensities}

WM hyperintensities (WMH) were identified on T2-weighted scans by an experienced observer blinded to subjects' identity, using a local thresholding segmentation technique (Jim Version 8.0, Xinapse Systems Ltd, Colchester, UK, http://www.xinapse.com), obtaining a ROI file for each scan. Then, for each subject, total WMH volume was obtained. The 4D WMH lesion masks were obtained using the following steps: (1) a WMH mask was created for each subject using Jim; (2) T2-weighted images were coregistered to the preprocessed DW MRI image using FMRIB's Linear Image Registration Tool (FLIRT) obtaining t2_to_DW image; (3) the previous registration was used to align each WMH mask with the DW space; (4) WMH masks were then coregistered to FMRIB standard space, obtaining a single 4D image including the spatial distribution of WMHs of patients and healthy controls. A visual quality check at each step was performed by an experienced observer blinded to subjects' identity. Subsequently, 4D WMH masks were coregistered to USCLobes brain atlas (http://brainsuite.org/ usclobes-description) and, for each lobe, we calculated the total number of voxels marked as part of a lesion for each group. To be consistent with the eLORETA-based analyses (i.e., CSD and LLC analyses), we grouped the lobes into six macro-areas (bilateral frontal, temporal, and parieto-occipital areas). Insula, cingulate and corpus callosum regions were included in the frontal area. Brainstem and cerebellum were not included in the analysis.

\section{Statistical analysis}

\section{Aim 1. Cross-sectional analyses}

Differences between patients and healthy controls were assessed at baseline. Clinical-demographic variables, cognitive scores, EEG (IAF and regional CSD) and MRI (brain total, GM and WM volumes, and WMH total and regional volumes) data were compared between groups using Fisher's exact test and non-parametric Wilcoxon signed-rank test (Bonferroni-corrected for multiple comparisons, $p<0.05, \mathrm{R}$ Software) for categorical and continuous variables, respectively. Network-Based Statistics (NBS) [33] were performed to identify regional LLC alterations in COVID-19 patients at the level of significance $p<0.05$ (Matlab Software). A corrected $p$ value was calculated using an age- and sexadjusted permutation analysis (10.000 permutations). Only frequencies found significantly different between groups in at least one EEG ROI at CSD and/or LLC analyses were selected and employed in subsequent analyses (Aim 2, see next paragraph).

Additionally, all possible correlations among baseline clinical, cognitive, EEG (IAF, CSD and LLC) and MRI (brain total, GM and WM volumes, and WMH total and regional volumes) data of patients were assessed. Correlation analyses were estimated using Spearman's coefficient adjusted for age, sex, and education (Bonferroni-corrected for multiple comparisons, $p<0.05$, R Software).

\section{Aim 2. Longitudinal analyses}

Changes over time of cognitive and EEG data in patients were assessed using linear mixed-effects models (Bonferroni-corrected for multiple comparisons, $p<0.05$, R Software). Time, age, sex, education, and individual follow-up duration were considered as fixed effects. Random effect of subject (ID) for each model was considered. Only the cognitive and EEG variables showing a significant change were considered into the following subgroup analyses (Aim 3 , see next paragraph).

Correlation analyses between cognitive and EEG data of patients at follow-up were estimated using Spearman's coefficient adjusted for age, sex, education and individual follow-up duration (Bonferroni-corrected for multiple comparisons, $p<0.05$, R Software). Linear regression models were implemented to investigate whether clinical, cognitive, EEG, and MRI data at baseline were predictive of cognitive and EEG data at follow-up in patients. Only the correlations resulting significant from the previous analyses were considered in the prediction models. The models included age, sex, education and individual follow-up duration. R2 goodness of fit statistic was estimated for each model at issue, for all patients at follow-up ( $p<0.05$, R Software).

\section{Aim 3. Subgroup analyses}

Dysgeusia and hyposmia have been proposed as possible hallmarks of SARS-CoV-2 cerebral spreading and have been associated with the development of cognitive impairment after COVID-19 [16, 34, 35]. Accordingly, we investigated cognitive and EEG changes over time in the two subgroups of patients with or without 
both dysgeusia and hyposmia during the acute phase of COVID-19 (socio-demographic and clinical variables are reported in Table e-4). A group-by-time interaction was explored using linear mixed-effects models (Bonferronicorrected for multiple comparisons, $p<0.05$, R Software). Time, age, sex, education, individual follow-up duration and the considered variable (cognitive or EEG) at baseline were included as fixed effects. Random effect of subject (ID) for each model was considered. Possible confounding clinical-demographic variables were explored using Fisher's exact test and ANOVA models (Bonferroni-corrected for multiple comparisons, $p<0.05$, $\mathrm{R}$ Software). Significantly different variables (or with a certain trend towards significance) between the two subgroups of patients were added into the models (Table e-4).

\section{Results}

\section{Aim 1. Cross-sectional analyses}

\section{Clinical-demographic data}

Socio-demographic and clinical features of patients and healthy controls who entered the cognitive, EEG and MRI analyses are reported in Table 1. Among study subjects, the most common chronic pathologies were hypertension, dyslipidemia and diabetes type 2; in particular, the patient group was characterized by a higher prevalence of hypertension than healthy controls only within the cognitive study. During the acute phase of COVID-19, $85.7 \%$ of patients were treated as inpatients, $26.5 \%$ and $4.1 \%$ of them required non-invasive mechanical ventilation (NIMV) and orotracheal intubation, respectively (Table 2). Moreover, neurological manifestations were reported for $73.5 \%$ of patients, with dysgeusia and
Table 1 Socio-demographics of study subjects at baseline (grouped in cognitive, EEG and MRI studies)

\begin{tabular}{|c|c|c|c|c|}
\hline & & Healthy controls & Patients & $p$ \\
\hline \multirow[t]{3}{*}{$\mathrm{N}$} & Cognitive & 36 & 49 & - \\
\hline & EEG & 33 & 49 & - \\
\hline & MRI & 36 & 36 & - \\
\hline \multirow[t]{3}{*}{$\operatorname{Sex}[M / F]$} & Cognitive & $20 / 16$ & $36 / 13$ & 0.46 \\
\hline & EEG & $22 / 11$ & $36 / 13$ & 0.62 \\
\hline & MRI & $20 / 16$ & $25 / 11$ & 0.55 \\
\hline \multirow[t]{3}{*}{ Age [years] } & Cognitive & $56.9 \pm 13.6$ & $60.8 \pm 12.6$ & 0.22 \\
\hline & EEG & $62.2 \pm 14.4$ & $60.8 \pm 12.6$ & 0.50 \\
\hline & MRI & $56.9 \pm 13.6$ & $58.5 \pm 13.3$ & 0.75 \\
\hline \multirow[t]{3}{*}{ Education [years] } & Cognitive & $12.1 \pm 4.2$ & $11.1 \pm 3.9$ & 0.29 \\
\hline & EEG & - & $11.1 \pm 3.9$ & - \\
\hline & MRI & $12.1 \pm 4.2$ & $11.5 \pm 3.9$ & 0.58 \\
\hline \multirow[t]{3}{*}{ Time between acquisitions [months] } & Cognitive & - & $8.2 \pm 0.9$ & - \\
\hline & EEG & - & $8.2 \pm 0.9$ & - \\
\hline & MRI & - & - & - \\
\hline \multirow{15}{*}{$\begin{array}{l}\text { Cardiovascular risk factors: } \\
\text { Hypertension } \\
\text { Smoke } \\
\text { Dyslipidemia } \\
\text { Diabetes mellitus } \\
\text { Obesity }\end{array}$} & \multirow[t]{5}{*}{ Cognitive } & $8 / 36(22.2)$ & $27 / 49(55.1)$ & 0.004 \\
\hline & & $8 / 36(22.2)$ & $11 / 49(22.4)$ & 1.00 \\
\hline & & $13 / 36(36.1)$ & $9 / 49$ (18.4) & 0.08 \\
\hline & & $1 / 36(2.8)$ & $6 / 49(12.2)$ & 0.23 \\
\hline & & $4 / 36(11.1)$ & $5 / 49(10.2)$ & 1.00 \\
\hline & \multirow[t]{5}{*}{ EEG } & $17 / 33(51.5)$ & $27 / 49(55.1)$ & 0.48 \\
\hline & & $4 / 33(12.1)$ & $11 / 49(22.4)$ & 0.47 \\
\hline & & $4 / 33(12.1)$ & $6 / 49(12.2)$ & 0.51 \\
\hline & & $2 / 33(6.1)$ & 9/49 (18.4) & 0.62 \\
\hline & & $2 / 33(6.1)$ & $5 / 49(10.2)$ & 0.70 \\
\hline & \multirow[t]{5}{*}{ MRI } & $8 / 36(22.2)$ & $15 / 36(41.7)$ & 0.13 \\
\hline & & $8 / 36(22.2)$ & $5 / 36(13.9)$ & 0.54 \\
\hline & & $13 / 36(36.1)$ & $8 / 36(22.2)$ & 0.30 \\
\hline & & $1 / 36(2.8)$ & 4/36 (11.1) & 0.36 \\
\hline & & 4/36 (11.1) & $2 / 36(5.6)$ & 0.67 \\
\hline
\end{tabular}

Values denote mean \pm standard deviation or number (percentage)

$P$ values refer to Fisher's exact test and non-parametric Wilcoxon signed-rank test (Bonferroni-corrected for multiple comparisons, $p<0.05$ ) for categorical and continuous variables, respectively 
Table 2 General clinical and neurological features of patients during the acute phase of COVID-19 (grouped in cognitive/EEG and MRI studies)

Acute phase clinical features of COVID-19 patients

\begin{tabular}{|c|c|c|}
\hline \multirow[t]{5}{*}{ Cognitive/EEG } & NEWS at ER admission & $5.9 \pm 3.2$ \\
\hline & Treated as inpatients & $42 / 49(85.7)$ \\
\hline & Treated with NIMV & $13 / 49(26.5)$ \\
\hline & Intensive care unit & $2 / 49(4.1)$ \\
\hline & Hospital stay [days] & $13.0 \pm 8.6$ \\
\hline \multirow[t]{5}{*}{ MRI } & NEWS at ER admission & $5.0 \pm 2.9$ \\
\hline & Treated as inpatients & $31 / 36(86.1)$ \\
\hline & Treated with NIMV & $9 / 36(25.0)$ \\
\hline & Intensive care unit & $1 / 36(2.8)$ \\
\hline & Hospital stay [days] & $12.6 \pm 8.6$ \\
\hline \multicolumn{3}{|c|}{ Acute phase neurologic manifestations of COVID-19 patients } \\
\hline \multirow[t]{4}{*}{ Cognitive/EEG } & Neurologic symptoms & $36 / 49(73.5)$ \\
\hline & Dysgeusia/hyposmia & $22 / 49(44.9)$ \\
\hline & Headache & $13 / 49(26.5)$ \\
\hline & Confusion/drowsiness & $11 / 49(22.4)$ \\
\hline \multirow[t]{4}{*}{ MRI } & Neurologic symptoms & $28 / 36(77.8)$ \\
\hline & Dysgeusia/hyposmia & $16 / 36(44.4)$ \\
\hline & Headache & $12 / 36(33.3)$ \\
\hline & Confusion/drowsiness & $9 / 36(25.0)$ \\
\hline
\end{tabular}

Values denote mean \pm standard deviation or number (percentage)

ER emergency room, NEWS National Early Warning Severity, NIMV non-invasive mechanical ventilation

hyposmia affecting $59.2 \%$ and $44.9 \%$ of them, respectively (Table 2). At baseline, acute neurological symptoms were resolved, but $26.5 \%$ of patients (13/49) reported asthenia.

\section{Cognitive study}

At baseline, $53 \%$ of patients showed an impairment in at least one cognitive domain, with a main involvement of the executive functions (Fig. 1A). In particular, 16\%, 6\% and $6 \%$ of patients showed a pure executive, memory and visual-spatial impairment, respectively, and $25 \%$ of subjects showed a multidomain impairment (with the $23 \%$ involving, among others, the executive domain). Moreover, $28 \%$ of patients presented with psychopathological disturbances (10\% depressive symptoms, 12\% PTSD features, 6\% both) (Fig. 1B). Concerning the comparison with healthy controls, patients performed worse in all investigated domains (Table 3).

\section{EEG study}

COVID-19 patients were characterized by a lower IAF than healthy subjects (Fig. 2, Table e-5). Patients also showed a greater CSD at delta frequency band in bilateral frontal and central-temporal regions when compared to healthy controls (Fig. 2, Table e-5). The connectivity analysis showed significant higher LLC values at delta band in patients when compared to healthy controls between all pairs of ROI (Table e-6). No other significant differences were found. According to these findings, only delta frequency band was selected for further analyses.

\section{MRI study}

No significant differences were found between COVID-19 patients and healthy subjects in terms of total brain, GM and WM volumes (Table 4). Total, right frontal and right parieto-occipital WMH volumes were greater in patients when compared to controls, whereas no significant differences were detected in other regions (Table 4).

\section{Correlation analysis}

Significant correlation findings at baseline are reported in Figure e-1. When considering the relationship between baseline clinical and cognitive data, lower performances at SDMT were related to more severe respiratory distress as assessed by NEWS at the admittance in the ER $(\rho=-0.43$, $p=0.03$ ). When analyzing the correlation between baseline cognitive and EEG data, better performances at FAB and at TMT-BA were related with greater right and left central-temporal CSD at delta frequency band ( $\rho$ ranging from 
Fig. 1 A The prevalence of cognitive deficits and $(\mathbf{B})$ of depressive and PTSD symptoms in COVID-19 patients, at both time points. Data are reported at baseline (2 months after hospital discharge) and at follow-up (10 month after hospital discharge). PTSD Post-traumatic stress disorder
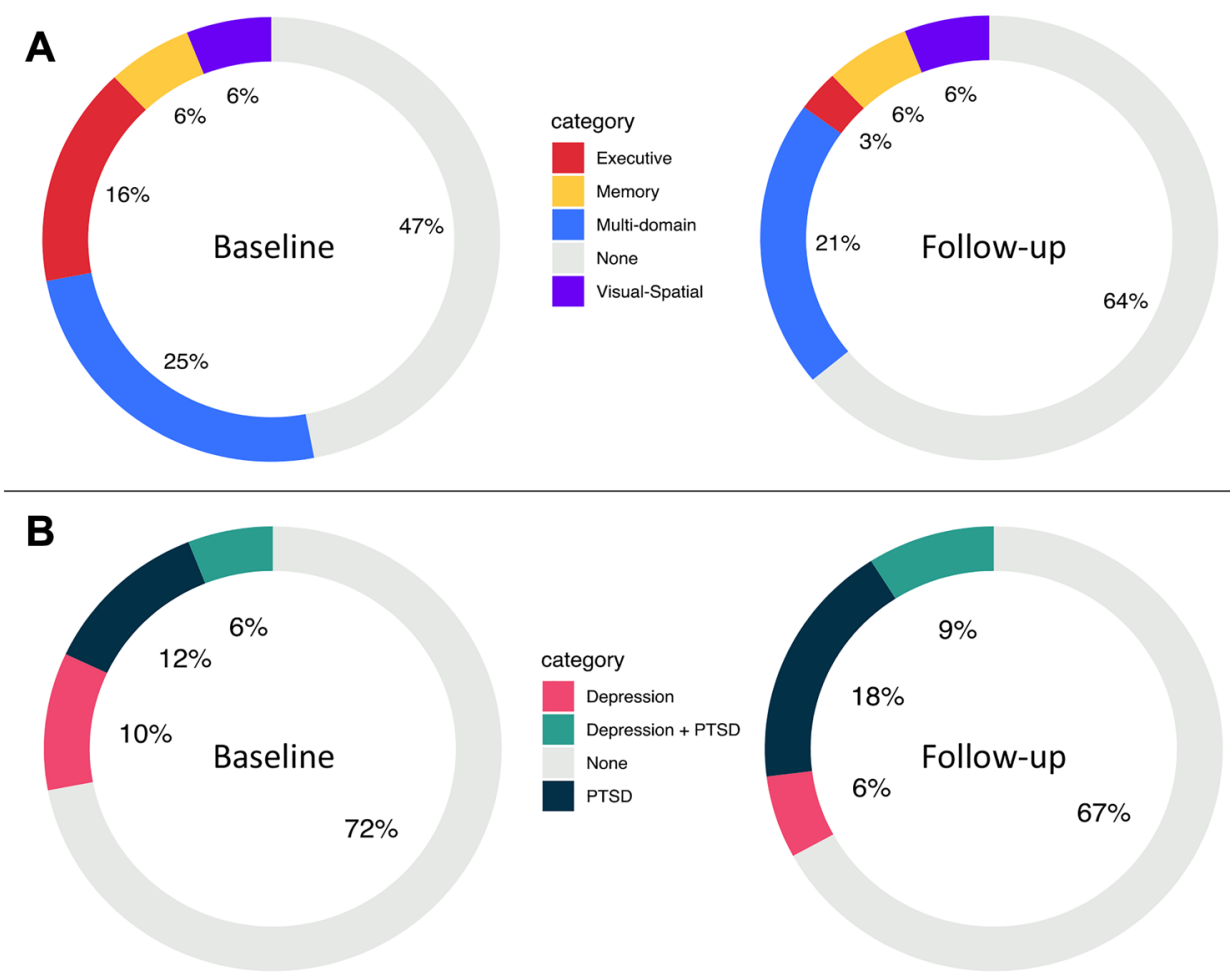

-0.41 to $0.45, p<0.05)$. Better performances at FAB were also associated with higher LLC values at delta frequency band between left parieto-occipital region and right frontal region $(\rho=0.42, p=0.045)$ and between left parietooccipital region and right central-temporal region $(\rho=0.44$, $p=0.02$ ). Correlating baseline clinical and MRI data, a greater WMH volume within the left frontal region was related to a higher frequency of cardiovascular risk factors in COVID-19 patients $(\rho=0.50, p=0.02)$. When exploring the relationship between baseline cognitive and MRI data, lower performances at RAVLT-immediate and -delayed recall were related to a higher WMH volume in the left parieto-occipital region ( $\rho$ ranging from -0.54 to $-0.51, p=0.01$ ).). No other significant correlations were observed.

\section{Aim 2. Longitudinal analyses}

\section{Clinical-demographic data}

Only 33 patients underwent longitudinal assessments (mean age $=60.6 \pm 12.9$, sex $=25$ male and 8 female, mean education $=11.3 \pm 3.9$ ). At follow-up no new-onset major neurological manifestations were reported, but $18.2 \%$ of patients (6/33) reported the persistence of asthenia.

\section{Cognitive study}

At follow-up, $36 \%$ of patients showed an impairment in at least one cognitive domain (Fig. 1A). In particular, 3\%, 6\% and $6 \%$ of patients showed a pure executive, memory and visual-spatial impairment, respectively, and $21 \%$ of subjects showed a multidomain impairment (with the $18 \%$ involving, among others, the executive domain). Moreover, $33 \%$ of patients still presented with psychopathological symptoms (6\% depressive, 18\% PTSD, 9\% both) (Fig. 1B). The linear mixed-effect models revealed a significant improvement of phonemic fluency test, SDMT, and RAVLT-immediate recall scores (Fig. 3A, Table 3). No significant changes were observed in the other cognitive tests.

\section{EEG study}

IAF of COVID-19 patients at follow-up was significantly increased compared with baseline (Fig. 3B, Table e-5). Analysis of CSD at delta frequency band showed no significant changes over time (Table e-5). Concerning connectivity analysis, a reduction of LLC values between left frontal and left parieto-occipital regions, between right frontal and left central-temporal regions, and between right frontal and right central-temporal regions was observed over time (Table e-6).

\section{Correlation and prediction analysis}

When exploring correlations with cognitive data at follow-up, better performances at TMT-BA were related to higher baseline left central-temporal delta CSD values ( $\rho=-0.55, p=0.002)$; a similar relationship, almost significant, was observed with delta CSD findings at baseline 
Table 3 Cognitive features of healthy controls and COVID-19 patients at the two time points

\begin{tabular}{|c|c|c|c|c|c|}
\hline & $\begin{array}{l}\text { Controls } \\
\text { baseline }\end{array}$ & $\begin{array}{l}\text { Patients } \\
\text { baseline }\end{array}$ & $\begin{array}{l}p \\
\text { controls vs patients }\end{array}$ & $\begin{array}{l}\text { Patients } \\
\text { follow-up }\end{array}$ & $\begin{array}{l}p \\
\text { for linear } \\
\text { trend } \\
\text { Patients }\end{array}$ \\
\hline $\mathrm{N}$ & 36 & 49 & & 33 & \\
\hline \multicolumn{6}{|l|}{ Global cognition } \\
\hline MMSE & $29.4 \pm 0.7$ & $27.7 \pm 1.9$ & $<0.001$ & $28.1 \pm 1.7$ & 1.00 \\
\hline \multicolumn{6}{|l|}{ Executive functions } \\
\hline Frontal assessment battery & - & $12.5 \pm 2.5$ & - & $14.5 \pm 2.2$ & 0.51 \\
\hline Symbol Digit Modalities Test & - & $35.1 \pm 1.9$ & - & $41.8 \pm 1.3$ & 0.01 \\
\hline Digit span, backward & $4.7 \pm 1.2$ & $3.4 \pm 1.5$ & $<0.001$ & $3.7 \pm 1.2$ & 1.00 \\
\hline TMT-A & $33.5 \pm 11.5$ & $50.3 \pm 25.3$ & $<0.001$ & $42.6 \pm 21.0$ & 0.41 \\
\hline TMT-BA & $70.8 \pm 44.5$ & $75.4 \pm 54.7$ & 0.93 & $75.9 \pm 53.6$ & 1.00 \\
\hline Cognitive estimations & - & $4.4 \pm 0.8$ & - & $4.7 \pm 0.5$ & 0.57 \\
\hline Phonemic fluency & $36.3 \pm 8.2$ & $27.8 \pm 10.2$ & 0.001 & $31.9 \pm 11.4$ & 0.01 \\
\hline \multicolumn{6}{|l|}{ Memory } \\
\hline Digit span, forward & $6.0 \pm 1.0$ & $5.1 \pm 0.7$ & $<0.001$ & $4.9 \pm 0.8$ & 1.00 \\
\hline RAVLT, immediate recall & $49.7 \pm 8.2$ & $29.3 \pm 9.4$ & $<0.001$ & $35.8 \pm 11.2$ & $<0.001$ \\
\hline RAVLT, delay recall & $11.4 \pm 2.4$ & $5.9 \pm 2.9$ & $<0.001$ & $7.1 \pm 3.3$ & 0.08 \\
\hline Complex figure*, recall & $0.76 \pm 0.14$ & $0.4 \pm 2.4$ & $<0.001$ & $0.4 \pm 0.1$ & 0.55 \\
\hline \multicolumn{6}{|l|}{ Visuospatial functions } \\
\hline Complex figure*, copy & $0.92 \pm 0.03$ & $0.8 \pm 0.1$ & $<0.001$ & $0.8 \pm 0.1$ & 1.00 \\
\hline VOSP, Position discrimination & - & $7.8 \pm 2.8$ & - & $7.5 \pm 2.9$ & 1.00 \\
\hline VOSP, Cube analysis & - & $8.1 \pm 2.5$ & - & $8.8 \pm 1.6$ & 1.00 \\
\hline \multicolumn{6}{|l|}{ Language } \\
\hline SAND, Sentence comprehension & - & $7.6 \pm 0.9$ & - & $7.8 \pm 0.5$ & 1.00 \\
\hline SAND, Word comprehension & - & $11.9 \pm 0.6$ & - & $11.8 \pm 0.5$ & 1.00 \\
\hline SAND, Word comprehension, living & - & $6.0 \pm 0.1$ & - & $5.9 \pm 0.2$ & 1.00 \\
\hline SAND, Word comprehension, non-living & - & $5.9 \pm 0.5$ & - & $5.9 \pm 0.3$ & 1.00 \\
\hline SAND, Naming & - & $13.1 \pm 1.5$ & - & $13.7 \pm 0.7$ & 0.51 \\
\hline SAND, Naming, living & - & $6.6 \pm 0.8$ & - & $6.9 \pm 0.3$ & 0.24 \\
\hline SAND, Naming, non-living & - & $6.5 \pm 0.9$ & - & $6.7 \pm 0.5$ & 1.00 \\
\hline
\end{tabular}

Neuropsychological values are expressed as raw scores

Values denote mean \pm standard deviation or number (percentage)

MMSE Mini-mental state examination, RAVLT Rey auditory verbal learning test, SAND Screening for aphasia in neurodegeneration, TMT Trail making test, VOSP Visual object and space perception battery

*Rey's or Benson's figure. $P$ values in the fourth column refer to Wilcoxon signed-rank test (Bonferroni-corrected for multiple comparisons, $p<0.05)$ when comparing healthy subjects and patients at baseline. $P$ values in the sixth column refer to linear mixed-effect models adjusted for age, sex, education and individual follow-up duration (Bonferroni-corrected for multiple comparisons, $p<0.05$ ) when exploring changes in cognitive performances of patients over time

in the contralateral regions $(\rho=-0.48, p=0.051)$. No other significant correlations were observed. Linear regression models built on the results of previous correlation analyses at follow-up showed that higher delta CSD values at baseline in left $\left(R^{2}=0.42, p=0.002\right)$ and right $\left(R^{2}=0.33, p=0.01\right)$ central-temporal regions predicted better TMT-BA performances at follow-up.

\section{Aim 3. Subgroup analyses}

Age, sex, education, and clinical and global cognition data did not differ between controls and both patient subgroups (i.e., those with and without dysgeusia/hyposmia in the acute phase) and between the two subgroups of patients (Table e-4). The presence of both PTSD and depression showed a 


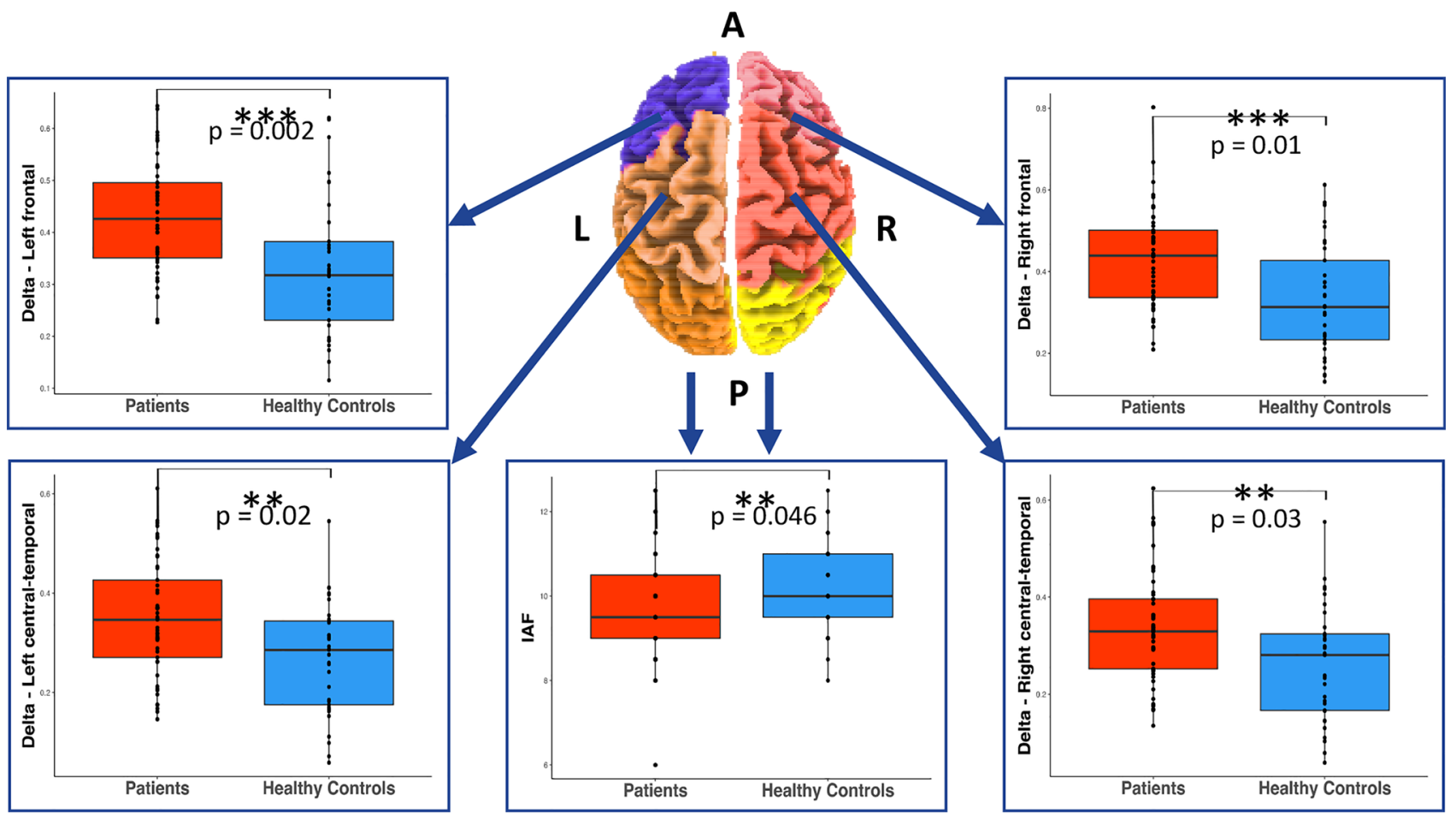

Fig. 2 Current source density analysis at delta frequency band and IAF analysis at baseline. $P$ values refer to Wilcoxon signed-rank test (Bonferroni-corrected for multiple comparisons, $p<0.05$ ) between

Table 4 MRI features of healthy controls and COVID-19 patients at baseline

\begin{tabular}{llll}
\hline & Controls & $\begin{array}{l}\text { Patients } \\
\text { b }\end{array}$ & \multicolumn{2}{l}{$\begin{array}{l}p \\
\text { baseline }\end{array}$} & baseline & controls vs patients \\
\hline $\mathrm{N}$ & 36 & 36 & \\
Brain volumes $\left(\mathrm{cm}^{3}\right)$ & & & \\
GM volume & $794 \pm 48$ & $772 \pm 54$ & 0.15 \\
WM volume & $682 \pm 44$ & $667 \pm 37$ & 0.13 \\
Total brain volume & $1476 \pm 80$ & $1440 \pm 81$ & 0.10 \\
White matter hyperintensity volumes $\left(\mathrm{cm}^{3}\right)$ & \\
Total & $0.52 \pm 1.44$ & $2.06 \pm 4.41$ & 0.01 \\
Left frontal & $0.16 \pm 0.37$ & $0.52 \pm 1.12$ & 0.35 \\
Right frontal & $0.14 \pm 0.39$ & $0.59 \pm 1.16$ & $<0.001$ \\
Left temporal & $0.01 \pm 0.03$ & $0.03 \pm 0.09$ & 0.91 \\
Right temporal & $0.02 \pm 0.06$ & $0.03 \pm 0.08$ & 1.00 \\
Left parieto-occip- & $0.10 \pm 0.38$ & $0.45 \pm 1.03$ & 0.45 \\
$\quad$ ital & & & \\
Right parieto- & $0.08 \pm 0.29$ & $0.40 \pm 1.04$ & 0.03 \\
$\quad$ occipital & & & \\
\hline
\end{tabular}

Values denote mean \pm standard deviation or number (percentage)

$P$ values refer to Wilcoxon signed-rank test (Bonferroni-corrected for multiple comparisons, $p<0.05$ ) when comparing healthy subjects and patients at baseline

$G M$ gray matter, $W M$ white matter
COVID-19 patients and healthy controls. $A$ anterior, IAF Individual Alpha Frequency, $L$ left, $P$ posterior, $R$ right

certain trend towards significance, being more frequently found in patients with dysgeusia/hyposmia $(p=0.08)$. Therefore, this condition was inserted into the longitudinal models. Linear mixed-effect models showed only a different improvement at RAVLT-immediate recall over time between the two patient subgroups (Fig. 3A). Patients with dysgeusia/ hyposmia exhibited a lower improvement relative to patients without dysgeusia/hyposmia. No significant results were observed at phonemic fluency test, SDMT or IAF (Fig. 3).

\section{Discussion}

This is the first study exploring long-term neurocognitive and neurophysiological consequences on adult COVID-19 survivors up to 10 months after infection resolution with structured neuropsychological and resting-state EEG assessments. Our findings showed that $53 \%$ of patients had disturbances in at least one cognitive domain 2 months after COVID-19 resolution. At follow-up, a significant amelioration of memory and executive performances was observed in the whole group; despite this, cognitive deficits were still evident in $36 \%$ of patients. Moreover, about $30 \%$ of participants reported depressive and/or PTSD symptoms at baseline, still persisting at follow-up. 


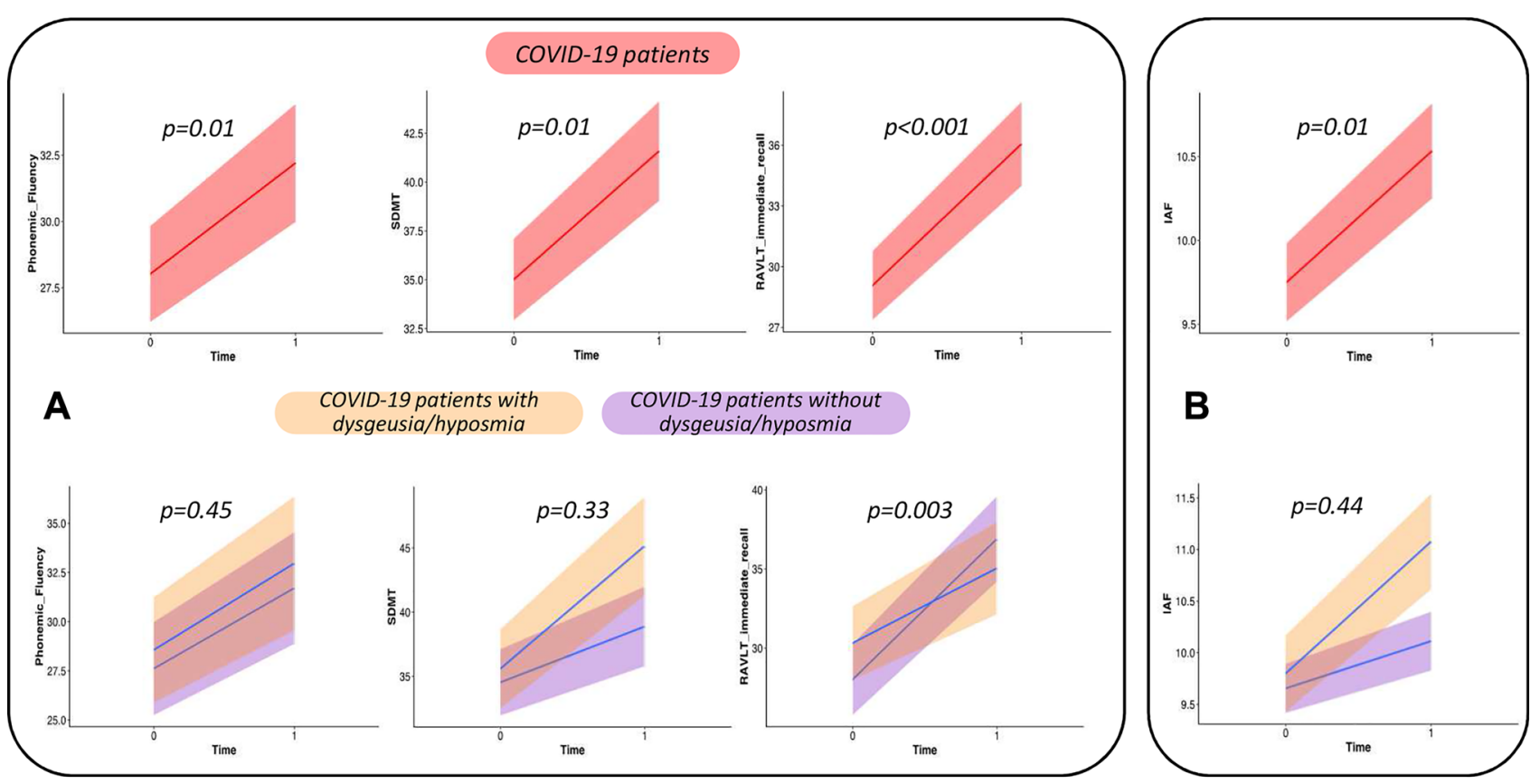

Fig. 3 Longitudinal and subgroup analyses investigating cognitive (A) and IAF (B) changes over time in COVID-19 patients. Upper row: $p$ values refer to linear mixed-effect models adjusted for age, sex, education and individual follow-up duration in the whole patients' group (Bonferroni-corrected for multiple comparisons, $p<0.05)$. Lower row: $p$ values refer to linear mixed-effect models

Albeit conditioned by the fact that study patients were selected on the basis of reported new-onset cognitive disturbances, our findings are in line with previous studies showing cognitive and/or psychiatric disturbances at different timepoints after COVID-19 infection resolution in the general population $[8,9,11,17]$. We found that executive functions (such as flexible thinking, planning and information processing) were the main involved cognitive domains at both baseline and follow-up. At baseline, the executive impairment (measured with the SDMT, reflecting speed of information processing) was related to the NEWS at ER admission, an index of respiratory distress, consistently with previous studies evaluating the association of acute illness severity and persistence of long-term COVID-19-related symptoms [12, 36, 37]. Intensive care and grade of oxygen need, in particular, are among factors that have been suggested as main drivers of such symptoms. Furthermore, previous evidence of the interrelatedness of acute pulmonary distress and cognitive and mental health shows similar results [37]. Episodes of pneumonia, for instance, are associated with increased risk of cognitive impairment in one-third of adults older than 65 and in approximately onefifth of younger patients $[37,38]$. Likewise, PTSD is a common finding in post-acute respiratory distress syndrome; in particular, longer lengths of intensive care unit stay and adjusted for age, sex, education, individual follow-up duration, the considered variable (cognitive or EEG) at baseline and the presence of both PTSD and depression in the two subgroups of patients with/ without dysgeusia/hyposmia (Bonferroni-corrected for multiple comparisons, $p<0.05)$. IAF individual alpha frequency, RAVLT Rey auditory verbal learning test, $S D M T$ symbol digit modality test

increased duration of both sedation and mechanical ventilation have been associated with PTSD [37, 39]. In our study, 12 out of 49 patients received intensive care or non-invasive ventilation and, among these, only 2 patients showed PTSD. Thus, at least in our sample, sedation and mechanical ventilation cannot be the cause of all PTSD symptoms that we observed.

An alternative explanation for mental disorders after COVID-19, and in particular for PTSD, might be searched in being clinically treated at home and in isolation. We were indeed surprised to observe that, in our cohort, executive dysfunctions and psychopathological disturbances were present in most of patients under 50 years of age. These patients were those clinically milder and that received medical care at home for most of the disease time, not requiring mechanical ventilation. Facing a disease like COVID-19 isolated at home, fearing possible complications in the absence of direct medical control and without a structured mental health intervention, could have had an impact on their psychiatric and cognitive functioning, as previously suggested [40].

Interestingly, patients complaining both dysgeusia and hyposmia during the acute illness showed a significantly lower improvement at verbal memory tests over time relative to patients without dysgeusia/hyposmia. Consistently, a recent MRI study on 394 patients with available scans 
before and after COVID-19, revealed a loss of GM within the limbic cortical areas directly linked to the olfactory and gustatory systems and involved in memory processes [16]. These findings suggest possible mechanisms for the spread of the disease (or the virus itself) within the brain. Among others, it was recently hypothesized that COVID-19 might work as a trigger on a pre-existing Alzheimer-like pathology by accelerating amyloid deposition [41]. Whatever the exact pathogenic pathways, our findings corroborate the hypothesis of an increased vulnerability of the structures involved in memory processes due to COVID-19.

COVID-19 patients also showed a greater WMH volume than controls, correlating with worse memory function at baseline and with the total number of cardiovascular risk factors, whereas no association with acute illness severity indices was detected (i.e., NEWS, mechanical ventilation need). Despite patients and healthy subjects were matched for risk factors number (Table 1), a trend towards a higher prevalence of hypertension within patients group was detected $(p=0.13)$, which could account for an increased risk for WMH formation already before COVID-19 [42], but also for worse infection severity [43], and subsequently for developing new lesions during the illness itself [44]. Differently from previous studies [16], we did not find a loss of brain total volume or of GM and WM volumes in COVID-19 survivors.

In this study, we did not explore neuroinflammation, which has been previously demonstrated to play a certain role in the development, maintenance or recovery of cognitive and neuropsychiatric symptoms $[8,11]$. Autopsy studies confirmed indeed a pronounced inflammation, with microglia activation, vascular activation and microthrombosis within the deep WM and brainstem of COVID19 patients $[17,45,46]$. Thus, neuropathology seems to indicate possible damages to the reticular activation system and to the cortical cholinergic projections from deep brain structures, which have been previously related to a tonic alpha rhythmic background activity in the healthy brain [46-48]. Such observations are consistent with the observed reduction of IAF and the increase of delta CSD of COVID-19 survivors at baseline EEG. Other harmful processes, for example hypoxic and metabolic changes that we previously related to acute-phase increase of frontal slow waves [18], could have contributed to follow-up findings, which, though, showed no relationship with acutephase severity indices. Unexpectedly, we found that higher delta CSD at baseline was related to better executive functions and predicted better cognitive outcomes at follow-up. Similarly, the patients that at baseline showed stronger connectivity in delta band also had better executive performances. Pathological cortical deafferentation, thus, does not entirely explain findings at delta band. We hypothesize that some sort of compensatory mechanisms might have been triggered in high-performing patients. Delta oscillations, especially in anterior regions of the brain, have been indeed related to a better performance in attention shifting and working memory tasks [49]. Consistently, we observed a significant positive correlation of delta CSD with TMT and FAB, which investigate the same skills. The increase of IAF within normality values and the reduction of delta LLC at follow-up, together with the concomitant amelioration at cognitive tests, might conversely suggest repairer mechanisms occurring over time.

Future studies exploring the possible differences in terms of clinical, psychopathological, EEG and MRI features between patients reversing to a normal cognitive state and those persistently impaired at follow-up will be crucial to disentangle the hallmarks of individual resilience.

Our study has some limitations. The sample size is relatively small and includes patients selected on the basis of reported new-onset cognitive disturbances and who do not represent then the general population of COVID-19 survivors. Moreover, we had no cognitive, EEG or MRI data previous to COVID-19, precluding us from estimating the exact impact of the illness on our findings. In addition, we did not investigate psychiatric disturbances through extended and structured assessments, but they were reported by patients to our experienced neuropsychologists. Furthermore, we did not perform a structured neurological evaluation at follow-up. Also, 19-channel EEG has a low spatial resolution and precluded the CSD and LLC analyses at sub-regional level. Furthermore, we did not acquire longitudinal MRI data, for which we could not explore possible changes of cerebral volumes over time. Finally, we could not enroll a control cohort with subacute respiratory dysfunction or viral infections different from COVID-19, reason why the specificity of results for SarsCoV-2 infection cannot be confirmed.

At 2 months after COVID-19 resolution, patients exhibited interrelated cognitive, EEG and cerebrovascular alterations, together with prominent psychiatric symptoms. Severity of acute illness was associated with executive dysfunction, but no association with EEG or MRI findings was detected. Longitudinal data at 10 months after hospital discharge showed an amelioration of cognitive and EEG findings, but also a persistence of some cognitive and of all psychiatric disturbances. Dysgeusia and hyposmia during acute COVID-19 were related with increased vulnerability in memory functions over time. Whether these alterations are directly linked with the infection itself or with its related consequences is still to be determined, as well as whether they are completely reversible or part of a neurodegenerative process.

Supplementary Information The online version contains supplementary material available at https://doi.org/10.1007/s00415-022-11047-5. 
Data availability The dataset used and analyzed during the current study will be made available by the corresponding author upon request to qualified researchers (i.e., affiliated to a university or research institution/hospital).

\section{Declarations}

Conflicts of interest G. Cecchetti, S. Basaia, A. Barbieri, R. Cardamone, M.P. Bernasconi, V. Castelnovo, C. Cividini, M. Cursi, M. Vabanesi, M. Impellizzeri, S.M. Lazzarin, G.F. Fanelli, F. Minicucci, G. Giacalone, A. Falini, M. Falautano, P. Rovere-Querini, L. Roveri have nothing to disclose. E. Canu receives or has received research supports form the Italian Ministry of Health. F. Agosta is Associate Editor of NeuroImage: clinical, has received speaker honoraria from Roche and Biogen Idec, and receives or has received research supports from the Italian Ministry of Health, AriSLA (Fondazione Italiana di Ricerca per la SLA), the European Research Council and Foundation Research on Alzheimer Disease. M. Filippi Editor-in-Chief of the Journal of Neurology and Associate Editor of Human Brain Mapping, Neurological Sciences, and Radiology; received compensation for consulting services and/or speaking activities from Almiral, Alexion, Bayer, Biogen, Celgene, Eli Lilly, Genzyme, Merck-Serono, Novartis, Roche, Sanofi, Takeda, and Teva Pharmaceutical Industries, and receives research support from Biogen Idec, Merck-Serono, Novartis, Roche, Teva Pharmaceutical Industries, Italian Ministry of Health, Fondazione Italiana Sclerosi Multipla, and ARiSLA (Fondazione Italiana di Ricerca per la SLA).

Ethical approval The study has been approved by the appropriate ethics committee and has, therefore, been performed in accordance with the ethical standards laid down in the 1964 Declaration of Helsinki and its later amendments. All participants provided written informed consent prior to study inclusion.

\section{References}

1. Parasher A (2021) COVID-19: current understanding of its pathophysiology, clinical presentation and treatment. Postgrad Med J 97:312-320

2. Nalbandian A, Sehgal K, Gupta A, Madhavan MV, McGroder C, Stevens JS, Cook JR, Nordvig AS, Shalev D, Sehrawat TS, Ahluwalia N, Bikdeli B, Dietz D, Der-Nigoghossian C, LiyanageDon N, Rosner GF, Bernstein EJ, Mohan S, Beckley AA, Seres DS, Choueiri TK, Uriel N, Ausiello JC, Accili D, Freedberg DE, Baldwin M, Schwartz A, Brodie D, Garcia CK, Elkind MSV, Connors JM, Bilezikian JP, Landry DW, Wan EY (2021) Post-acute COVID-19 syndrome. Nat Med 27:601-615

3. Taquet M, Geddes JR, Husain M, Luciano S, Harrison PJ (2021) 6-month neurological and psychiatric outcomes in 236379 survivors of COVID-19: a retrospective cohort study using electronic health records. Lancet Psychiatry 8:416-427

4. Vanderlind WM, Rabinovitz BB, Miao IY, Oberlin LE, BuenoCastellano C, Fridman C, Jaywant A, Kanellopoulos D (2021) A systematic review of neuropsychological and psychiatric sequalae of COVID-19: implications for treatment. Curr Opin Psychiatry $34: 420-433$

5. Raman B, Cassar MP, Tunnicliffe EM, Filippini N, Griffanti L, Alfaro-Almagro F, Okell T, Sheerin F, Xie C, Mahmod M, Mozes FE, Lewandowski AJ, Ohuma EO, Holdsworth D, Lamlum H, Woodman MJ, Krasopoulos C, Mills R, McConnell FAK, Wang C, Arthofer C, Lange FJ, Andersson J, Jenkinson M, Antoniades C, Channon KM, Shanmuganathan M, Ferreira VM, Piechnik SK,
Klenerman P, Brightling C, Talbot NP, Petousi N, Rahman NM, Ho LP, Saunders K, Geddes JR, Harrison PJ, Pattinson K, Rowland MJ, Angus BJ, Gleeson F, Pavlides M, Koychev I, Miller KL, Mackay C, Jezzard P, Smith SM, Neubauer S (2021) Mediumterm effects of SARS-CoV-2 infection on multiple vital organs, exercise capacity, cognition, quality of life and mental health, post-hospital discharge. EClin Med 31:100683

6. Woo MS, Malsy J, Pottgen J, Seddiq Zai S, Ufer F, Hadjilaou A, Schmiedel S, Addo MM, Gerloff C, Heesen C, Schulze Zur Wiesch J, Friese MA (2020) Frequent neurocognitive deficits after recovery from mild COVID-19. Brain Commun 2(2):fcaa205

7. Zhou H, Lu S, Chen J, Wei N, Wang D, Lyu H, Shi C, Hu S (2020) The landscape of cognitive function in recovered COVID19 patients. J Psychiatr Res 129:98-102

8. Mazza MG, Palladini M, De Lorenzo R, Magnaghi C, Poletti S, Furlan R, Ciceri F, Rovere-Querini P, Benedetti F, Group C-BOCS (2021) Persistent psychopathology and neurocognitive impairment in COVID-19 survivors: effect of inflammatory biomarkers at three-month follow-up. Brain Behav Immun 94:138-147

9. Del Brutto $\mathrm{OH}, \mathrm{Wu} \mathrm{S}$, Mera RM, Costa AF, Recalde BY, Issa NP (2021) Cognitive decline among individuals with history of mild symptomatic SARS-CoV-2 infection: a longitudinal prospective study nested to a population cohort. Eur J Neurol 28(10):3245-3253

10. Postolache TT, Benros ME, Brenner LA (2020) Targetable biological mechanisms implicated in emergent psychiatric conditions associated With SARS-CoV-2 infection. JAMA Psychiat. https:// doi.org/10.1001/jamapsychiatry.2020.2795

11. Mazza MG, De Lorenzo R, Conte C, Poletti S, Vai B, Bollettini I, Melloni EMT, Furlan R, Ciceri F, Rovere-Querini P, Benedetti F, Group C-BOCS (2020) Anxiety and depression in COVID19 survivors: role of inflammatory and clinical predictors. Brain Behav Immun 89:594-600

12. Huang C, Huang L, Wang Y, Li X, Ren L, Gu X, Kang L, Guo L, Liu M, Zhou X, Luo J, Huang Z, Tu S, Zhao Y, Chen L, Xu D, Li Y, Li C, Peng L, Li Y, Xie W, Cui D, Shang L, Fan G, Xu J, Wang G, Wang Y, Zhong J, Wang C, Wang J, Zhang D, Cao B (2021) 6-month consequences of COVID-19 in patients discharged from hospital: a cohort study. Lancet 397:220-232

13. McElvaney OJ, McEvoy NL, McElvaney OF, Carroll TP, Murphy MP, Dunlea DM, Ni Choileain O, Clarke J, O'Connor E, Hogan G, Ryan D, Sulaiman I, Gunaratnam C, Branagan P, O'Brien ME, Morgan RK, Costello RW, Hurley K, Walsh S, de Barra E, McNally C, McConkey S, Boland F, Galvin S, Kiernan F, O'Rourke J, Dwyer R, Power M, Geoghegan P, Larkin C, O'Leary RA, Freeman J, Gaffney A, Marsh B, Curley GF, McElvaney NG (2020) Characterization of the inflammatory response to severe COVID-19 Illness. Am J Respir Crit Care Med 202:812-821

14. Tang N, Li D, Wang X, Sun Z (2020) Abnormal coagulation parameters are associated with poor prognosis in patients with novel coronavirus pneumonia. J Thromb Haemost 18:844-847

15. Heneka MT, Golenbock D, Latz E, Morgan D, Brown R (2020) Immediate and long-term consequences of COVID-19 infections for the development of neurological disease. Alzheimers Res Ther 12:69

16. Douaud G, Lee S, Alfaro-Almagro F, Arthofer C, Wang C, Lange F, Andersson JLR, Griffanti L, Duff E, Jbabdi S, Taschler B, Winkler A, Nichols TE, Collins R, Matthews PM, Allen N, Miller KL, Smith SM (2021) Brain imaging before and after COVID-19 in UK Biobank. MedRxiv. https://doi.org/10.1101/2021.06.11.21258 690

17. Hosp JA, Dressing A, Blazhenets G, Bormann T, Rau A, Schwabenland M, Thurow J, Wagner D, Waller C, Niesen WD, Frings L, Urbach H, Prinz M, Weiller C, Schroeter N, Meyer PT (2021) Cognitive impairment and altered cerebral glucose metabolism in the subacute stage of COVID-19. Brain 144:1263-1276 
18. Cecchetti G, Vabanesi M, Chieffo R, Fanelli G, Minicucci F, Agosta F, Tresoldi M, Zangrillo A, Filippi M (2020) Cerebral involvement in COVID-19 is associated with metabolic and coagulation derangements: an EEG study. J Neurol 267:3130-3134

19. Petrescu AM, Taussig D, Bouilleret V (2020) Electroencephalogram (EEG) in COVID-19: a systematic retrospective study. Neurophysiol Clin 50:155-165

20. Antony AR, Haneef Z (2020) Systematic review of EEG findings in 617 patients diagnosed with COVID-19. Seizure 83:234-241

21. Kostakis I, Smith GB, Prytherch D, Meredith P, Price C, Chauhan A, Investigating PACF, C, (2021) The performance of the National Early Warning Score and National Early Warning Score 2 in hospitalised patients infected by the severe acute respiratory syndrome coronavirus 2 (SARS-CoV-2). Resuscitation 159:150-157

22. Quinn TJ, Dawson J, Walters M (2008) Dr John Rankin; his life, legacy and the 50th anniversary of the Rankin stroke scale. Scott Med J 53:44-47

23. van Swieten JC, Koudstaal PJ, Visser MC, Schouten HJ, van Gijn J (1988) Interobserver agreement for the assessment of handicap in stroke patients. Stroke 19:604-607

24. Homan RW, Herman J, Purdy P (1987) Cerebral location of international 10-20 system electrode placement. Electroencephalogr Clin Neurophysiol 66:376-382

25. Caso F, Cursi M, Magnani G, Fanelli G, Falautano M, Comi G, Leocani L, Minicucci F (2012) Quantitative EEG and LORETA: valuable tools in discerning FTD from AD? Neurobiol Aging 33:2343-2356

26. Cecchetti G, Agosta F, Basaia S, Cividini C, Cursi M, Santangelo R, Caso F, Minicucci F, Magnani G, Filippi M (2021) Restingstate electroencephalographic biomarkers of alzheimer's disease. Neuroimage Clin 31:102711

27. Delorme A, Makeig S (2004) EEGLAB: an open source toolbox for analysis of single-trial EEG dynamics including independent component analysis. J Neurosci Methods 134:9-21

28. Makeig S, Westerfield M, Jung TP, Covington J, Townsend J, Sejnowski TJ, Courchesne E (1999) Functionally independent components of the late positive event-related potential during visual spatial attention. J Neurosci 19:2665-2680

29. Pascual-Marqui RD (2007) Discrete, 3D distributed, linear imaging methods of electric neuronal activity. Part 1: exact, zero error localization. arXiv:0710.3341 [math-ph], 2007-October-17. http:// arxiv.org/pdf/0710.3341

30. Pascual-Marqui RD (2007) Instantaneous and lagged measurements of linear and nonlinear dependence between groups of multivariate time series: frequency decomposition. arXiv:0711.1455 [stat.ME], 2007-November-09. http://arxiv.org/abs/0711.1455

31. Smith SM, De Stefano N, Jenkinson M, Matthews PM (2001) Normalized accurate measurement of longitudinal brain change. J Comput Assist Tomogr 25:466-475

32. Smith SM, Zhang Y, Jenkinson M, Chen J, Matthews PM, Federico A, De Stefano N (2002) Accurate, robust, and automated longitudinal and cross-sectional brain change analysis. Neuroimage 17:479-489

33. Zalesky A, Fornito A, Bullmore ET (2010) Network-based statistic: identifying differences in brain networks. Neuroimage 53:1197-1207

34. Al-Sarraj S, Troakes C, Hanley B, Osborn M, Richardson MP, Hotopf M, Bullmore E, Everall IP (2021) Invited review: the spectrum of neuropathology in COVID-19. Neuropathol Appl Neurobiol 47:3-16

35. Pirker-Kees A, Platho-Elwischger K, Hafner S, Redlich K, Baumgartner C (2021) Hyposmia Is associated with reduced cognitive function in COVID-19: first preliminary results. Dement Geriatr Cogn Disord 50:68-73

36. Arnold DT, Hamilton FW, Milne A, Morley AJ, Viner J, Attwood M, Noel A, Gunning S, Hatrick J, Hamilton S, Elvers KT, Hyams C, Bibby A, Moran E, Adamali HI, Dodd JW, Maskell NA, Barratt SL (2021) Patient outcomes after hospitalisation with COVID-19 and implications for follow-up: results from a prospective UK cohort. Thorax 76:399-401

37. Riordan P, Stika M, Goldberg J, Drzewiecki M (2020) COVID19 and clinical neuropsychology: a review of neuropsychological literature on acute and chronic pulmonary disease. Clin Neuropsychol 34:1480-1497

38. Girard TD, Self WH, Edwards KM, Grijalva CG, Zhu Y, Williams DJ, Jain S, Jackson JC (2018) Long-term cognitive impairment after hospitalization for community-acquired pneumonia: a prospective cohort study. J Gen Intern Med 33:929-935

39. Davydow DS, Katon WJ, Zatzick DF (2009) Psychiatric morbidity and functional impairments in survivors of burns, traumatic injuries, and ICU stays for other critical illnesses: a review of the literature. Int Rev Psychiatry 21:531-538

40. Mohamed AE, Yousef AM (2021) Depressive, anxiety, and posttraumatic stress symptoms affecting hospitalized and home-isolated COVID-19 patients: a comparative cross-sectional study. Middle East Curr Psychiatry 28:28

41. Rahman MA, Islam K, Rahman S, Alamin M (2021) Neurobiochemical Cross-talk between COVID-19 and alzheimer's disease. Mol Neurobiol 58:1017-1023

42. van Dijk EJ, Breteler MM, Schmidt R, Berger K, Nilsson LG, Oudkerk M, Pajak A, Sans S, de Ridder M, Dufouil C, Fuhrer R, Giampaoli S, Launer LJ, Hofman A, Consortium C (2004) The association between blood pressure, hypertension, and cerebral white matter lesions: cardiovascular determinants of dementia study. Hypertension 44:625-630

43. Du Y, Zhou N, Zha W, Lv Y (2021) Hypertension is a clinically important risk factor for critical illness and mortality in COVID19: a meta-analysis. Nutr Metab Cardiovasc Dis 31:745-755

44. Fraiman P, Godeiro Junior C, Moro E, Cavallieri F, Zedde M (2020) COVID-19 and cerebrovascular diseases: a systematic review and perspectives for stroke management. Front Neurol 11:574694

45. Matschke J, Lutgehetmann M, Hagel C, Sperhake JP, Schroder AS, Edler C, Mushumba H, Fitzek A, Allweiss L, Dandri M, Dottermusch M, Heinemann A, Pfefferle S, Schwabenland M, Sumner Magruder D, Bonn S, Prinz M, Gerloff C, Puschel K, Krasemann S, Aepfelbacher M, Glatzel M (2020) Neuropathology of patients with COVID-19 in Germany: a post-mortem case series. Lancet Neurol 19:919-929

46. Yong SJ (2021) Persistent brainstem dysfunction in long-COVID: a hypothesis. ACS Chem Neurosci 12:573-580

47. Ricceri L, Minghetti L, Moles A, Popoli P, Confaloni A, De Simone R, Piscopo P, Scattoni ML, di Luca M, Calamandrei G (2004) Cognitive and neurological deficits induced by early and prolonged basal forebrain cholinergic hypofunction in rats. Exp Neurol 189:162-172

48. Osipova D, Ahveninen J, Kaakkola S, Jaaskelainen IP, Huttunen J, Pekkonen E (2003) Effects of scopolamine on MEG spectral power and coherence in elderly subjects. Clin Neurophysiol 114:1902-1907

49. Harmony T (2013) The functional significance of delta oscillations in cognitive processing. Front Integr Neurosci 7:83 